\title{
Potential use of visible and near-infrared spectroscopy for the analysis and diagnosis of chronic fatigue syndrome (Review)
}

\author{
AKIKAZU SAKUDO ${ }^{1,2}$ \\ ${ }^{1}$ Laboratory of Biometabolic Chemistry, School of Health Sciences, Faculty of Medicine, University of The Ryukyus, \\ Nishihara, Okinawa 903-0215; ${ }^{2}$ Department of Virology, Center for Infectious Disease Control, \\ Research Institute for Microbial Diseases, Osaka University, Yamadaoka, Suita, Osaka 565-0871, Japan
}

Received August 11, 2015; Accepted June 30, 2016

DOI: $10.3892 / \mathrm{mmr} .2016 .5476$

\begin{abstract}
At present, chronic fatigue syndrome (CFS) is diagnosed on the basis of clinical symptoms. Although various psychological, endocrinological and immunological abnormalities of patients with CFS have been reported, no clear consensus exists regarding the symptoms for this disorder. Thus, an objective diagnostic method for CFS is urgently required. The present study investigated the diagnosis and analysis of CFS using visible and near-infrared (Vis-NIR) spectroscopy. Previous studies have demonstrated the potential of Vis-NIR spectroscopy for diagnosing CFS by analyzing either serum samples as an invasive approach or thumbs as a non-invasive approach. Analysis of the Vis-NIR spectra of blood and thumbs suggested that factors absorbing in this spectral region are altered in patients with CFS compared with healthy individuals. These findings are likely to facilitate the search for biomarkers associated with CFS and to increase our understanding of the pathophysiology of the disorder. The current review aimed to outline the latest studies and discuss the future perspectives for CFS made possible by Vis-NIR spectroscopy.
\end{abstract}

\section{Contents}

1. Current status of chronic fatigue syndrome

2. CFS abnormalities and research

3. Vis-NIR spectroscopy for CFS research

4. Conclusion

Correspondence to: Dr Akikazu Sakudo, Laboratory of Biometabolic Chemistry, School of Health Sciences, Faculty of Medicine, University of The Ryukyus, 207 Uehara, Nishihara, Okinawa 903-0215, Japan

E-mail: sakudo@med.u-ryukyu.ac.jp

Key words: chronic fatigue syndrome, diagnosis, infrared spectroscopy, thumb, visible and near-infrared spectroscopy

\section{Current status of chronic fatigue syndrome}

Chronic fatigue syndrome (CFS) is a disabling condition in which the patient is affected by long-term fatigue. Symptoms, which can lasts for $\geq 6$ months, include tiredness, pain, breathing problems, depression leading to digestive disturbances, low-grade fever, difficulty in concentrating, and weakness of the immune system and muscles (1). One characteristic of this disease is that the symptoms are not relieved by increased rest (1). Another characteristic of CFS is the absence of intervention or medication that is universally effective in treating the symptoms (2). Furthermore, CFS causes not only personal problems, but also economic problems. Several previous studies have demonstrated that the incidence of CFS is $0.4 \%$ in the USA and other countries (3) and $0.26 \%$ in Japan (4). CFS is present in 522/100,000 females and 291/100,000 males in the USA, indicating that CFS incidence in females is higher compared with in males (2). In addition, economic losses due to CFS are estimated to be as high as $\$ 9.1$ billion per annum in the USA (5) and $¥ 408$ billion per annum in Japan (4). Therefore, CFS has an impact on society in general, as well as on the individual patient.

While patients affected by CFS sometimes suffer from recognized symptoms, they often experience other social problems, which makes the disorder difficult to recognize. Research performed by the Centers for Disease Control and Prevention (Atlanta, GA, USA) estimates that $<20 \%$ of patients with CFS in the USA have been successfully diagnosed $(3,6)$. Clearly, the establishment of a reliable diagnostic test for CFS will improve our understanding of this disorder. However, several obstacles are preventing the accomplishment of this goal. One such obstacle is the high degree of heterogeneity in the symptoms of patients with CFS (7). Thus, at present, CFS is diagnosed on the basis of the presenting symptoms, coupled with the exclusion of other medical disorders (1). The other predominant barriers to diagnosing patients with CFS are an absence of biophysical and biochemical signs that identify the disease, and a lack of diagnostic laboratory tests (7). Consequently, typical screening results of blood samples for CFS are often unrevealing or negative. As a result, CFS diagnosis requires experience and techniques that can be performed only by skilled doctors on the basis of exclusion of all other possible causes of chronic fatigue as listed. 
A

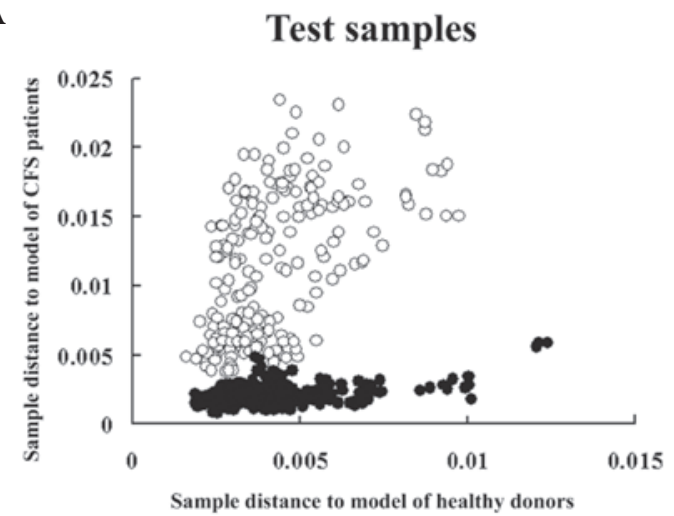

B

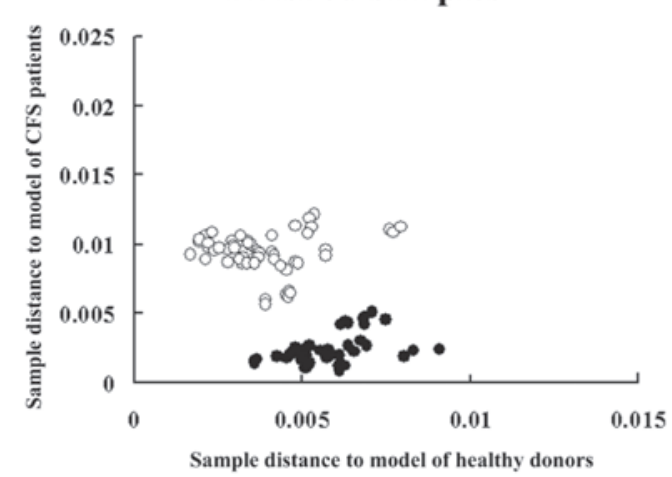

C

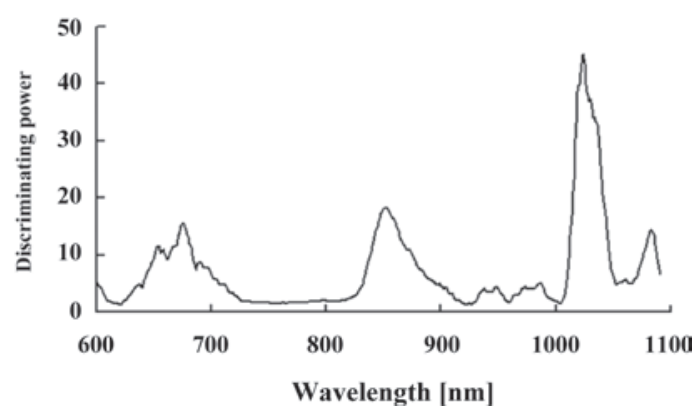

Figure 1. Vis-NIR spectroscopic analysis of sera for the diagnosis of CFS A blood sample was obtained from the antecubital vein of CFS patients and healthy donors. Sera from 77 patients with CFS and 71 healthy donors were used as test samples to develop a calibration model for diagnosis. A further 99 samples were masked and used for prediction. The Vis-NIR spectral data of the test samples were pre-processed and subjected to SIMCA (8) calibration modeling to develop a multivariate model to diagnose CFS Pirouette software (version 3.11; Infometrics, Woodinville, WA, USA) was employed for spectral data processing. The Coomans plot (9), which plots class distances against each other, demonstrated that the healthy donor class (open circles) and CFS patient class (closed circles) of (A) test samples and (B) masked samples did not share multivariate space. (C) The discriminating power of the SIMCA calibration model indicates important wavelengths for differentiating Vis-NIR spectra between CFS patients and healthy donors. Modified from Fig. 2 in Sakudo et al (10) with permission from Elsevier. Vis-NIR, visible and near-infrared spectroscopy; CFS, chronic fatigue syndrome; SIMCA, soft independent modeling of class analogy.

\section{CFS abnormalities and research}

Despite various data on the psychological, endocrinological and immunological abnormalities observed among CFS

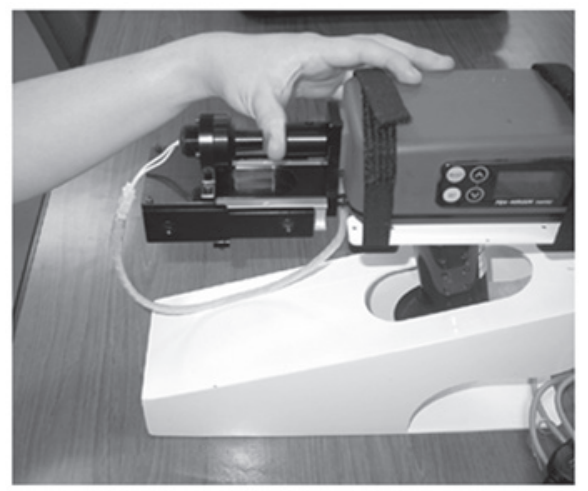

Figure 2. Spectrophotometer used for collecting Vis-NIR spectra from thumbs. Transmittance Vis-NIR spectra in the wavelength range 600-1,100 nm were non-invasively measured using an FQA-NIRGUN instrument (Japan Fantec Research Institute, Shizuoka, Japan). Reproduced from Fig. 1 in Sakudo et al (11) with permission from Elsevier. Vis-NIR, visible and near-infrared spectroscopy.

patients, no clear consensus exists on the symptoms for this disorder (12). Several indications of CFS have been reported, including altered levels of cytokines (13), immunoglobulins (13), autoantibodies (14), RNase L (15), 2'-5'oligo-adenylate synthetase (15), melatonin (16), dehydroepiandrostenedione (16), growth hormones (17), acylcarnitine (18), folic acid (19), vitamins (20), amino acids (20), carnitine Coenzyme Q10 (20), fatty acids (20) and minerals $(20,21)$. Altered cell populations and activity of the immune system have also been reported $(13,22)$. In addition, alterations in T-cell phenotype and proliferative response, along with the specific signature of the NK cell phenotype, have been reported in certain individuals with CFS (23). Other homeostatic changes involving the opioid system (24) and arginine vasopressin system (24) may be associated with CFS. Furthermore, adrenocorticotropic hormone and the cortisol response appear to be aberrant among patients with CFS (16).

The severe levels of fatigue and disability associated with CFS may be associated with peripheral inflammation and immune activation of blood cells, as is the case with neuroinflammatory and autoimmune illnesses (25). The mental and physical fatigue associated with CFS appears to be the consequence of interactions between multiple systemic and central pathways that take place via immune-inflammatory and neuroinflammatory networks (26). Such interactions would be supported by the activation of cytokines and immune cells, which has been reported in numerous previous studies $(27,28)$.

This background to CFS prompted us to investigate fundamental abnormalities in patients with CFS and to develop an objective diagnostic method for this disease. As a result, our research group has recently explored an approach based on visible and near-infrared (Vis-NIR) spectroscopy.

\section{Vis-NIR spectroscopy for CFS research}

The analysis of Vis-NIR spectra from the sera of patients with CFS identified certain characteristics that could be distinguished from the corresponding spectra of healthy 
A

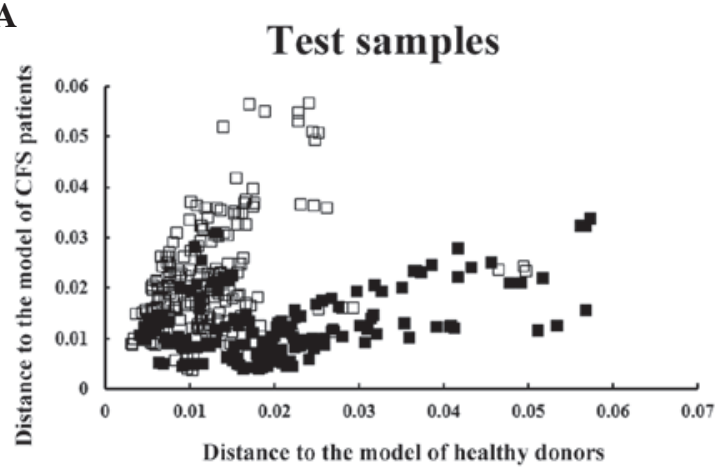

B

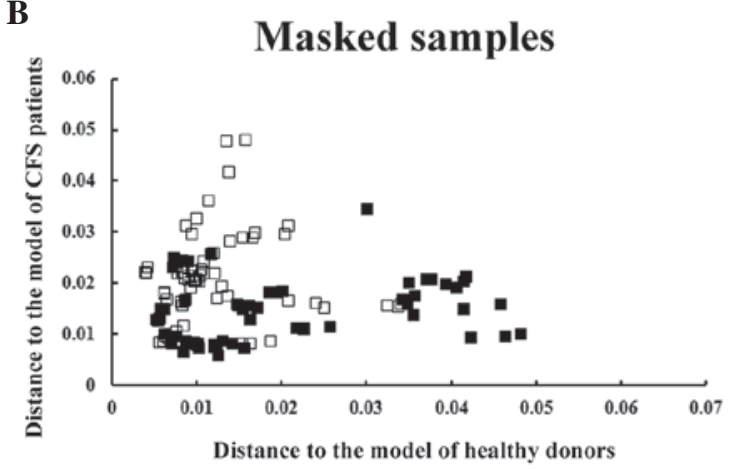

C Discriminating power

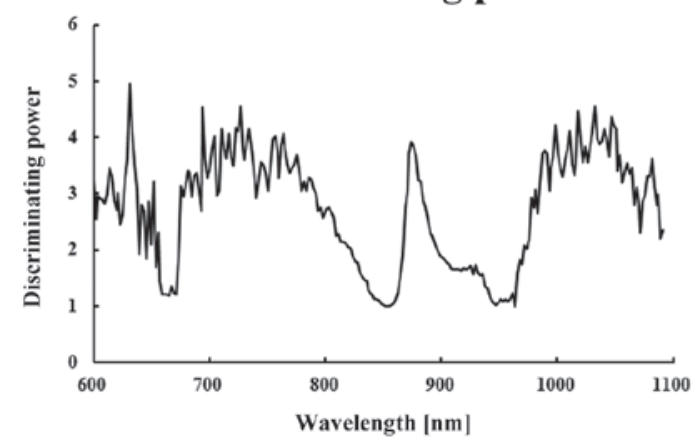

Figure 3. SIMCA of Vis-NIR calibration and prediction of CFS using thumb spectra. Vis-NIR spectral data obtained from the thumbs of healthy volunteers and CFS patients were pre-processed and subjected to SIMCA calibration modeling to develop a multivariate model to diagnose CFS. The Coomans plot of SIMCA demonstrated that the healthy volunteer class (open squares) and CFS patient class (closed squares) of (A) test samples and (B) masked samples did not share multivariate space. (C) Discriminating power of the SIMCA calibration model. Modified from Fig. 3 in Sakudo et al (11) with permission from Elsevier. Vis-NIR, visible and near-infrared spectroscopy; CFS, chronic fatigue syndrome.

donors $(12,29)$. A chemometrics analysis, termed soft independent modeling of class analogy (SIMCA), was applied to develop a multivariate model to discriminate between the Vis-NIR spectra of patients with CFS and those of healthy individuals (Fig. 1). As a result, the SIMCA models correctly predicted 54/54 (100\%) healthy subjects and 42/45 (93.3\%) patients with CFS based on the analysis of Vis-NIR spectra from masked serum samples (10).

Subsequently, to develop a non-invasive approach, Vis-NIR spectroscopic analysis of thumbs was performed (Fig. 2). The SIMCA models were applied to the Vis-NIR spectra of thumbs from patients with CFS and healthy individuals
Table I. Spectroscopic comparison of the ratio of oxyhemoglobin to deoxyhemoglobin in the thumbs of patients with CFS and healthy controls.

\begin{tabular}{lcc}
\hline & \multicolumn{2}{c}{ Absorbance at $850-760 \mathrm{~nm}$} \\
\cline { 2 - 3 } Characteristic & Male & Female \\
\hline Healthy & $0.6137 \pm 0.0073$ & $0.5005 \pm 0.0082$ \\
CFS & $0.6517 \pm 0.0108$ & $0.5352 \pm 0.0080$ \\
D'Agostino-pearson test & Yes & Yes \\
(parametric data or not) & & \\
Unpaired t test (P-value) & $(0.0027)^{\mathrm{a}}$ & $(0.0027)^{\mathrm{a}}$ \\
$\begin{array}{l}\text { Mann-Whitney test } \\
\text { (P-value) }\end{array}$ & - & - \\
\hline
\end{tabular}

The mean Vis-NIR spectra of patients with CFS and healthy controls showed sharp peaks at 694,970 and $1,060 \mathrm{~nm}$, and broad peaks in the regions of $740-760$ and $830-850 \mathrm{~nm}$. The broad peak at $740-760 \mathrm{~nm}$ is associated with deoxyhemoglobin (30), whereas that at $830-850 \mathrm{~nm}$ is associated with oxyhemoglobin (31) and the oxidation status of cytochrome $c$ oxidase $(31,32)$. Therefore, the ratio of absorbance at 850 to that at $760 \mathrm{~nm}$ in smoothing- and standard normal variate-corrected Vis-NIR spectra was compared in males and females (69 healthy males, 42 CFS males, 53 healthy females and 61 CFS females). An increased ratio of oxyhemoglobin to deoxyhemoglobin in CFS thumbs was observed in the two groups. Absorbance is shown as the average \pm standard error of the mean. ${ }^{a} \mathrm{P}<0.01$. Reproduced from Table III in Sakudo et al (33) with permission from Elsevier. CFS, chronic fatigue syndrome; Vis-NIR, visible and near-infrared spectroscopy.

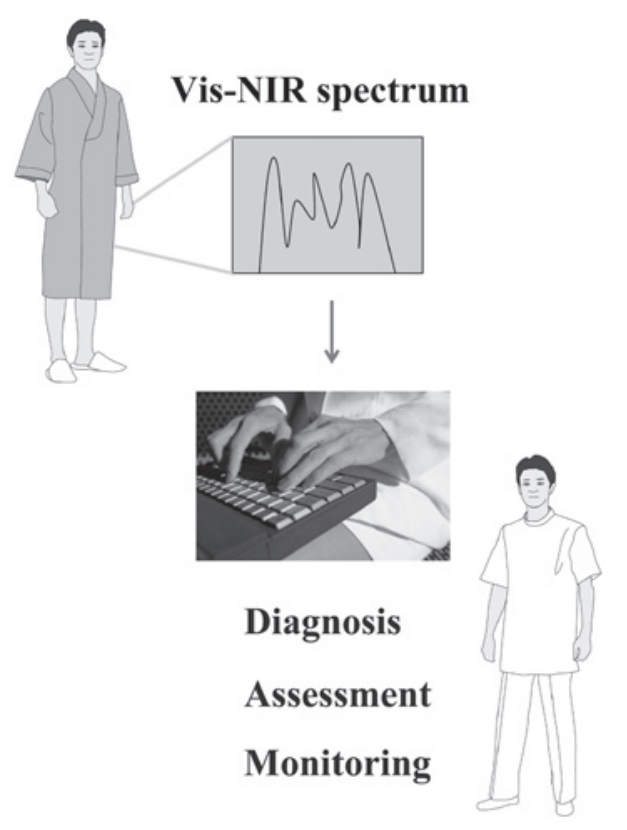

Figure 4. Future perspectives of Vis-NIR spectroscopy for CFS. Currently, CFS can be diagnosed on the basis of clinical symptoms only by skilled doctors. Thus, clinician experience and specialist techniques are required for the diagnosis of this condition. Vis-NIR spectroscopy may facilitate an objective and rapid diagnosis for CFS. In addition to diagnosis, Vis-NIR spectroscopy may also contribute to disease monitoring and assessment. To enable diagnosis, assessment and monitoring of CFS by Vis-NIR spectroscopy, the construction of a robust model by chemometrics analysis of Vis-NIR spectra will be important. Vis-NIR, visible and near-infrared spectroscopy; CFS, chronic fatigue syndrome. 
(Fig. 3). The model successfully predicted 51/60 (83.3\%) healthy subjects and 42/60 (70\%) patients with CFS (11).

The discriminating power of the calibration models from these previous studies suggested the presence of common factors among the sera and thumbs of patients with CFS (Figs. 1C and 3C). One of these factors may be associated with blood flow and energy metabolism, since the Vis-NIR spectra of thumbs suggested that patients with CFS have a significantly higher oxyhemoglobin content (Table I), and significantly increased oxidation of heme $a+a_{3}$ and copper in cytochrome $c$ oxidase (33).

IR spectroscopy is a form of vibrational spectroscopy. As such, the principal of IR spectroscopy is similar to that of Vis-NIR spectroscopy (34), although there are certain differences. The absorption observed in IR is due to fewer overtones as compared with Vis-NIR, resulting in sharper bands with higher intensity. Thus, IR spectroscopy is a powerful tool for CFS research. A previous study using IR spectroscopy found that fingernails of patients with CFS showed a decrease in $\alpha$-helix content and increased $\beta$-sheet content compared with those of healthy individuals, suggesting reduced levels of normal protein elements of the nail plate (35).

\section{Conclusion}

As described above, previous studies have demonstrated the potential of Vis-NIR spectroscopy for the diagnosis of CFS using serum samples and thumbs. Furthermore, analysis of the Vis-NIR and IR spectra of sera, thumbs and fingernails suggests that factors absorbing in this spectral region are altered in patients with CFS. Combining chemometric analysis of spectra obtained from CFS samples with analysis of a spectra database may enable us to identify potential candidates for CFS biomarkers. Currently, although vast IR spectra databases, including the KnowItAll Informatics System (Bio-Rad Laboratories, Inc., Philadelphia, PA, USA), are commercially available, no similarly large NIR spectra databases exist. Thus, construction of an NIR spectra database would be necessary for a chemometrics-based study. Further analysis using such a spectra database would facilitate the search for biomarkers for CFS and aid our understanding of the pathophysiology of this disorder.

Although the current review has focused on previous studies using Vis-NIR spectroscopy to examine CFS, this approach can be applied to other diseases, including cancer $(36,37)$, diabetes $(38,39)$, Alzheimer's disease (40) and epilepsy $(41,42)$. Therefore, the application of Vis-NIR spectroscopy to the diagnosis and analysis of disease in general is likely to increase. Its applications include not only diagnosis, but also assessment and monitoring of diseases (Fig. 4). In addition, Vis-NIR spectroscopy coupled with quantitative chemometrics analyses, including partial least squares and principal component regression, can be used to predict levels of biochemical constituents, including triglycerides, cholesterols, urea and lactate in body fluids, including blood $(43,44)$ and hematocrit in the body $(45)$. Therefore, this method may contribute to advances in clinical laboratory testing as well as maintaining human health in the form of a home testing kit.

\section{Acknowledgements}

The present study was supported by Grant-in-Aids for Scientific Research from the Japan Society for the Promotion of Science.

\section{References}

1. Fukuda K, Straus SE, Hickie I, Sharpe MC, Dobbins JG and Komaroff A: The chronic fatigue syndrome: A comprehensive approach to its definition and study. International chronic fatigue syndrome study group. Ann Intern Med 121: 953-959, 1994.

2. Bested AC, Logan AC and Howe R: Hope and Help for Chronic Fatigue Syndrome and Fibromyalgia. Cumberland House, Nashville, TN, 2008.

3. Jason LA, Richman JA, Rademaker AW, Jordan KM, Plioplys AV, Taylor RR, McCready W, Huang CF and Plioplys S: A community-based study of chronic fatigue syndrome. Arch Intern Med 159: 2129-2137, 1999.

4. Kuratsune H: Overview of chronic fatigue syndrome focusing on prevalence and diagnostic criteria. Nihon Rinsho 65: 983-990, 2007 (In Japanese).

5. Reynolds KJ, Vernon SD, Bouchery E and Reeves WC: The economic impact of chronic fatigue syndrome. Cost Eff Resour Alloc 2: 4, 2004

6. Reyes M, Nisenbaum R, Hoaglin DC, Unger ER, Emmons C, Randall B, Stewart JA, Abbey S, Jones JF, Gantz N, et al: Prevalence and incidence of chronic fatigue syndrome in Wichita, Kansas. Arch Intern Med 163: 1530-1536, 2003.

7. Vernon SD, Whistler T, Aslakson E, Rajeevan M and Reeves WC: Challenges for molecular profiling of chronic fatigue syndrome. Pharmacogenomics 7: 211-218, 2006.

8. Wold S: Pattern Recognition by means of disjoint principal components models. Pattern Recognition 8: 127-139, 1976.

9. Coomans D, Broeckaert I, Derde MP, Tassin A, Massart DL and Wold S: Use of a microcomputer for the definition of multivariate confidence regions in medical diagnosis based on clinical laboratory profiles. Comput Biomed Res 17: 1-14, 1984.

10. Sakudo A, Kuratsune H, Kobayashi T, Tajima S, Watanabe Y and Ikuta K: Spectroscopic diagnosis of chronic fatigue syndrome by visible and near-infrared spectroscopy in serum samples. Biochem Biophys Res Commun 345: 1513-1516, 2006.

11. Sakudo A, Kuratsune H, Kato YH and Ikuta K: Visible and near-infrared spectra collected from the thumbs of patients with chronic fatigue syndrome for diagnosis. Clin Chim Acta 413: $1629-1632,2012$.

12. Sakudo A, Hakariya Y, Kobayashi T, Sugimoto A and Ikuta K: Possible application of visible and near-infrared spectral patterns in serum to provide emerging clue to biomarkers for chronic fatigue syndrome. J IiME 2: 4-7, 2008.

13. Patarca R: Cytokines and chronic fatigue syndrome. Ann NY Acad Sci 933: 185-200, 2001.

14. Vernon SD and Reeves WC: Evaluation of autoantibodies to common and neuronal cell antigens in Chronic Fatigue Syndrome. J Autoimmune Dis 2: 5, 2005.

15. Nijs $J$ and De Meirleir K: Impairments of the 2-5A synthetase/RNase L pathway in chronic fatigue syndrome. In vivo 19: 1013-1021, 2005.

16. Cleare AJ: The neuroendocrinology of chronic fatigue syndrome. Endocr Rev 24: 236-252, 2003.

17. Allain TJ, Bearn JA, Coskeran P, Jones J, Checkley A, Butler J, Wessely S and Miell JP: Changes in growth hormone, insulin, insulinlike growth factors (IGFs), and IGF-binding protein-1 in chronic fatigue syndrome. Biol Psychiatry 41: 567-573, 1997.

18. Kuratsune H, Yamaguti K, Takahashi M, Misaki H, Tagawa S and Kitani T: Acylcarnitine deficiency in chronic fatigue syndrome. Clin Infect Dis 18 (Suppl 1): S62-S67, 1994.

19. Jacobson W, Saich T, Borysiewicz LK, Behan WM, Behan PO and Wreghitt TG: Serum folate and chronic fatigue syndrome. Neurology 43: 2645-2647, 1993.

20. Werbach MR: Nutritional strategies for treating chronic fatigue syndrome. Altern Med Rev 5: 93-108, 2000.

21. Maes M, Mihaylova I and De Ruyter M: Lower serum zinc in Chronic Fatigue Syndrome (CFS): Relationships to immune dysfunctions and relevance for the oxidative stress status in CFS. J Affect Disord 90: 141-147, 2006. 
22. Whiteside TL and Friberg D: Natural killer cells and natural killer cell activity in chronic fatigue syndrome. Am J Med 105 (Suppl): 27S-34S, 1998.

23. Curriu M, Carrillo J, Massanella M, Rigau J, Alegre J, Puig J, Garcia-Quintana AM, Castro-Marrero J, Negredo E, Clotet B, et al: Screening NK-, B- and T-cell phenotype and function in patients suffering from Chronic Fatigue Syndrome. J Transl Med 11: 68, 2013.

24. Parker AJ, Wessely S and Cleare AJ: The neuroendocrinology of chronic fatigue syndrome and fibromyalgia. Psychol Med 31 : 1331-1345, 2001

25. Morris G, Anderson G, Galecki P, Berk M and Maes M: A narrative review on the similarities and dissimilarities between myalgic encephalomyelitis/chronic fatigue syndrome (ME/CFS) and sickness behavior. BMC Med 11: 64, 2013

26. Morris G, Berk M, Galecki P, Walder K and Maes M: The neuro-immune pathophysiology of central and peripheral fatigue in systemic immune-inflammatory and neuro-immune diseases. Mol Neurobiol 53: 1195-1219, 2016.

27. Dell'Osso L, Bazzichi L, Baroni S, Falaschi V, Conversano C, Carmassi C and Marazziti D: The inflammatory hypothesis of mood spectrum broadened to fibromyalgia and chronic fatigue syndrome. Clin Exp Rheumatol 33 (1 Suppl 88): S109-S116, 2015.

28. Nijs J, Nees A, Paul L, De Kooning M, Ickmans K, Meeus M and Van Oosterwijck J: Altered immune response to exercise in patients with chronic fatigue syndrome/myalgic encephalomyelitis: A systematic literature review. Exerc Immunol Rev 20: 94-116, 2014

29. Sakudo A, Hakariya Y, Kobayashi $T$ and Ikuta K: Visible and near-infrared (Vis-NIR) spectroscopy: Introduction and perspectives for diagnosis of chronic fatigue syndrome. J IiME 1 : 8-18, 2007.

30. Heise HM: Applications of near-infrared spectroscopy in medical sciences. In: Near-infrared Spectroscopy: Principles, Instruments, Applications. Siesler HW, Ozaki Y, Kawata S and Heise HM (eds.) Wiley-VCH, Weinheim, pp289-333, 2002.

31. Matsunaga A, Nomura Y, Kuroda S, Tamura M, Nishihira J and Yoshimura N: Energy-dependent redox state of heme a + a3 and copper of cytochrome oxidase in perfused rat brain in situ. Am J Physiol 275: C1022-C1030, 1998.

32. Hoshi Y, Hazeki O and Tamura M: The oxygen dependency of the redox state of heme and copper in cytochrome oxidase in vitro. Adv Exp Med Biol 248: 71-76, 1989.
33. Sakudo A, Kato YH, Tajima S, Kuratsune $\mathrm{H}$ and Ikuta K: Visible and near-infrared spectral changes in the thumb of patients with chronic fatigue syndrome. Clin Chim Acta 403: 163-166, 2009.

34. Stuart B: Biological Applications of Infrared Spectroscopy. John Wiley \& Sons Ltd., New York, 1997.

35. Sakudo A, Kuratsune H, Kato YH and Ikuta K: Secondary structural changes of proteins in fingernails of chronic fatigue syndrome patients from Fourier-transform infrared spectra. Clin Chim Acta 402: 75-78, 2009.

36. Simick MK, Jong R, Wilson B and Lilge L: Non-ionizing near-infrared radiation transillumination spectroscopy for breast tissue density and assessment of breast cancer risk. J Biomed Opt 9: 794-803, 2004.

37. Liu KZ, Shi M, Man A, Dembinski TC and Shaw AR: Quantitative determination of serum LDL cholesterol by near-infrared spectroscopy. Vib Spectrosc 38: 203-208, 2005.

38. Robinson MR, Eaton RP, Haaland DM, Koepp GW, Thomas EV, Stallard BR and Robinson PL: Noninvasive glucose monitoring in diabetic patients: A preliminary evaluation. Clin Chem 38: 1618-1622, 1992.

39. Gabriely I, Wozniak R, Mevorach M, Kaplan J, Aharon Y and Shamoon H: Transcutaneous glucose measurement using near-infrared spectroscopy during hypoglycemia. Diabetes Care 22: 2026-2032, 1999.

40. Hock C, Villringer K, Müller-Spahn F, Hofmann M, Schuh-Hofer S, Heekeren H, Wenzel R, Dirnagl U and Villringer A: Near infrared spectroscopy in the diagnosis of Alzheimer's disease. Ann N Y Acad Sci 777: 22-29, 1996.

41. Watanabe E, Nagahori Y and Mayanagi Y: Focus diagnosis of epilepsy using near-infrared spectroscopy. Epilepsia 43 (Suppl 9): 50-55, 2002.

42. Sokol DK, Markand ON, Daly EC,Luerssen TG and Malkoff MD: Near infrared spectroscopy (NIRS) distinguishes seizure types. Seizure 9: 323-327, 2000 .

43. Hazen KH, Arnold MA and Small GW: Measurement of glucose and other analytes in undiluted human serum with near-infrared transmission spectroscopy. Anal Chim Acta 371: 255-267, 1998.

44. Kobayashi T, Kato YH, Tsukamoto M, Ikuta K and Sakudo A: Portable visible and near-infrared spectrophotometer for triglyceride measurements. Int J Mol Med 23: 75-79, 2009.

45. Sakudo A, Kato YH, Kuratsune H and Ikuta K: Non-invasive prediction of hematocrit levels by portable visible and near-infrared spectrophotometer. Clin Chim Acta 408: 123-127, 2009. 